\title{
Kinematic anharmonicity of internal rotation of molecules
}

\author{
V.A. Bataev*, V.I. Pupyshev, and I.A. Godunov \\ Lomonosov Moscow State University, Faculty of Chemistry, 119991 Moscow, Russia,
}

\begin{abstract}
The methods of analysis the strongly coupled vibrations are proposed for a number of molecules of aromatic and heterocyclic carbonyl (and some others) compounds. The qualitative principles are formulated for molecular systems with a significant kinematic anharmonicity.
\end{abstract}

In our report, we demonstrate methods of analysis of the strongly coupled molecular vibrations and formulate quality criteria to search of molecular systems with significant kinematic anharmonicity. As illustrations the molecules of aromatic and heterocyclic carbonyl (and some other) compounds are chosen.

The special attention was focuses on interactions of the rotation of the carbonyl group with other non-planar vibrations. It is interesting, that for the systems considered the form of the potential energy surface (PES) do not demonstrate any essential interactions of vibrational modes. The observed interaction is primarily due to the kinematic anharmonicity, which is manifested in a relatively large amplitude of changes of the offdiagonal elements of the kinematic matrix in some regions of PES.

The detailed analysis of internal rotation was performed for the benzaldehyde $\left(\mathrm{C}_{6} \mathrm{H}_{5} \mathrm{CHO}\right)$ and furfural $\left(\mathrm{C}_{5} \mathrm{H}_{4} \mathrm{OCHO}\right)$ molecules in the ground electronic states by methods of quantum chemistry. For the systems under investigation, this is a complex task, that requires the methods more accurate than the standard one-dimensional (1D) approximation. Really, one may find, for example, essential differences (especially for benzaldehyde molecule) between the experimental and calculated values of the potential barriers to internal rotation. For the molecules mentioned it was found the non-trivial situation of significant kinematic (but not dynamic) interaction between torsional vibration and out-of-plane CHO deformation [1,2]. Taking into account this kinematic interaction one may find the good agreement between experimental and calculated energies of torsional and out-of-plane CHO deformation energy levels within the two-dimensional (2D) approximation for the vibrational problem, while disagreement in energies for traditional 1D approximations are essential. It should be noted, that in the case of benzaldehyde molecule such agreement is reached, provided correct errors in assignment of torsional transitions in the experimental vibrational spectrum [1]. In particular, the results presented explain the disagreement in high level nonempirical and simplified 1D-estimates of barriers to internal rotation.

\footnotetext{
${ }^{*}$ Corresponding author: v.a.bataev@gmail.com
} 
The features of the internal rotation were also studied for other molecular systems containing benzene or various heterocyclic rings and the functional group (-CHO, -CDO, $\mathrm{CFO}$ et al.). It is shown that in some of the systems examined the relationship of rotation of the functional groups associated with the ring, and non-planar vibrations is predominantly kinematic, and is determined mainly by geometric parameters of this group and the corresponding distribution of point masses. The dependence of the kinematic effects on the mass distribution within the benzene or heterocyclic ring is much weaker. In contrast to the benzaldehyde molecule, the $1 \mathrm{D}$ potential function of internal rotation for related molecule of benzoyl fluoride $\left(\mathrm{C}_{6} \mathrm{H}_{5} \mathrm{CFO}\right)$ calculated as the minimal energy path is quite reliable; as a result, the $1 \mathrm{D}$ and $2 \mathrm{D}$ energies of the torsional energy levels are practically the same. This example and the results of testing of the similar molecules $\mathrm{C}_{6} \mathrm{H}_{5}-\mathrm{CXY}$ demonstrate clearly that one may expect the significant kinematic interaction between torsional vibration and out-of-plane CXY deformation for the systems where the masses of atoms X and Y differ essentially, as it is in the benzaldehyde molecule, but not in the benzoyl fluoride molecule $[1]$.

The support from Russian Foundation for Basic Research is acknowledged (16-0300794a).

\section{References}

1. I.A. Godunov, V.A.Bataev, A.V. Abramenkov, V.I. Pupyshev, J. Phys. Chem. A 118, 10159 (2014)

2. V.A.Bataev, A.V. Abramenkov, V.I. Pupyshev, I.A. Godunov, Spectrochim. Acta Part A 161, 155 (2016) 